\title{
SOLAR RADIO EMISSIONS IN VIEW OF THE SOLAR ORBITER MISSION
}

\author{
M. Maksimovic*
}

\begin{abstract}
One of the science goals of the Solar Orbiter mission is to study the connectivity between the solar corona and the inner Heliosphere as close as from 0.3 AU. With this respect the study of Solar radio emissions produced energetic electrons either flare or shock accelerated will be of prime importance. I will first review some recent findings obtained with the help of Ulysses, WIND and Stereo observations. I will then present the expected capabilities of the Solar Orbiter instrumentation relevant to the discussed topic.
\end{abstract}

* LESIA, Observatoire de Paris, UMR CNRS 8109, 92195 Meudon, France 
